\title{
Efeito de dez semanas de treinamento intervalado de alta intensidade na composição corporal de idosos
}

Effect of ten weeks of high-intensity interval training on body composition in the elderly

\author{
Eduardo dos Santos Soares Monteiro' \\ Patrícia Clara Costa de Amorim² \\ Alan dos Santos Vieira Formiga ${ }^{2}$ \\ Jaqueline Ferreira dos Reis ${ }^{2}$ \\ Brenda Helen Melo Caldas ${ }^{2}$
}

\begin{abstract}
RESUMO
O estudo investigou os efeitos do treinamento de intervalado alta intensidade (TIAI), versos treinamento aeróbio continuo (TAC) sobre a composição corporal de idosos. Vinte idosos previamente ativos de acordo com IPAQ foram divididos em dois grupos, sendo 10 no grupo TAC, (66,6 66,5 anos) e 10 no grupo TIAI, $(65,5 \pm 5,3$ anos) submetidos a dez semanas de treinamento. As sessões de treinamento aconteceram na cicloergometria com intensidade de 85 a $90 \%$ da frequência cardíaca de reserva (FCRes) para TIAI, e de 60 a 80\% da FCRes para o TAC. Houve uma redução da área de gordura visceral $(\mathrm{p}=0,0195)$, e aumento da massa magra $(\mathrm{p}=0,054)$ para $\mathrm{o}$ grupo TIAI. As demais variáveis da composição corporal, não foram alteradas em nenhum dos grupos. Dez semanas de exercício intervalado de alta intensidade foi capaz de reduzir a área de gordura visceral e aumentar a massa magra de idosos. Novos estudos são recomendados para maiores esclarecimentos.
\end{abstract}

Palavras-chave: Exercício aeróbio continuo. Exercício intervalado de alta intensidade. Idosos.

\begin{abstract}
The study investigated the effects of high intensity interval training (TIAI), versus continuous aerobic training (TAC) on the body composition of the elderly. Twenty elderly previously active according to IPAQ were divided into two groups, 10 in the TAC group, (66.6 \pm 6.5 years) and 10 in the TIAI group, $(65.5 \pm 5.3$ years $)$ submitted to ten weeks training. The training sessions took place in cycle ergometry with an intensity of 85 to $90 \%$ of the reserve heart rate (FCRes) for TIAI, and from 60 to $80 \%$ of the FCRes for TAC. There was a reduction in the area of visceral fat $(\mathrm{p}=$ $0.0195)$, and an increase in lean mass $(\mathrm{p}=0.054)$ for the TIAI group. The other body composition variables were not changed in any of the groups. Ten weeks of high-intensity interval exercise was able to reduce the visceral fat area and increase the lean mass of the elderly. New studies are recommended for further clarification.
\end{abstract}

Keywords: Continuous aerobic exercise. High intensity interval exercise. Seniors.

\section{RESUMEN}

El estudio investigó los efectos del entrenamiento en intervalos de alta intensidad (TIAI), versus el entrenamiento aeróbico continuo (TAC) en la composición corporal de los ancianos. Veinte personas mayores previamente activas según IPAQ se dividieron en dos grupos, 10 en el grupo TAC $(66.6 \pm 6.5$ años) y 10 en el grupo TIAI $(65.5 \pm 5.3$ años) sometidos a diez semanas entrenamiento Las sesiones de entrenamiento se realizaron en cicloergometría con una intensidad del 85 al 90\% de la frecuencia cardíaca de reserva (FCRes) para TIAI, y del 60 al $80 \%$ de las FCRes para TAC. Hubo una reducción en el

área de grasa visceral $(\mathrm{p}=0.0195)$, y un aumento en la masa magra $(\mathrm{p}=0.054)$ para el grupo TIAI. Las otras variables de composición corporal no se modificaron en ninguno de los grupos. Diez semanas de ejercicio de intervalos de alta intensidad pudieron reducir el área de grasa visceral y aumentar la masa magra de los ancianos. Se recomiendan nuevos estudios para mayor aclaración. 
Palabras-clave: ejercicio aeróbico continuo. Ejercicio de intervalos de alta intensidad. Personas mayores.

\section{INTRODUÇÃO}

A perda de independência pode ocorrer pela diminuição da mobilidade, devido em boa parte, à diminuição da massa muscular, força e resistência aeróbia(1). Portanto, a prática da atividade física regular em idosos colabora para uma velhice saudável, diminuindo ou retardando as perdas inerentes ao processo do envelhecimento.

Recentemente, o treinamento intervalado de alta intensidade (TIAI) tem atraído a atenção como uma opção de exercício tempo - eficiente para melhorar a saúde cardiovascular e metabólica (2). Este método de treinamento levou a uma redução promissora de fatores de risco cardiometabólica em adolescentes e, assim, tornou-se uma abordagem interessante para melhorar a saúde dos jovens (Logan et al., 2014).

Além disso, o TIAI vem mostrado eficaz para a gestão do peso corporal em pacientes com excesso de peso e obesos (3), e tem efeito sobre a composição corporal e o peso corporal entre os indivíduos obesos sedentários em comparação com aqueles que estão ativos (4). Evidências ainda são necessárias para clarificar o efeito desse tipo de treinamento sobre a composição corporal, uma vez que a maioria dos estudos abordando esta temática é com adultos, crianças e adolescentes obesos $(5,6)$.Apesar das evidencias sobre o exercício intervalado de alta intensidade a estudos que avaliam o efeito desse treinamento em idosos, ainda são escassos. Diante disso, o presente estudo tem como objetivo, avaliar o efeito de 10 semanas de exercício intervalado de alta intensidade na composição corporal de idosos.

\section{MATERIAIS E MÉTODOS}

\section{Participantes}

Foram recrutados e avaliados 75 indivíduos entretanto, 40 se recusaram a comparecer ao treinamento e 15 faltaram a mais de $20 \%$ do treinamento, tornandose ilegíveis para a pesquisa. Deste modo, concluíram o estudo 20 idosos ativos de acordo com o Questionário Internacional de Atividade Física (IPAQ) (7) que foram randomicamente divididos em dois grupos: Grupo Treinamento Aeróbio contínuo (TAC) (idade 66,6 66,5 anos) e grupo Treinamento Intervalado de Alta Intensidade (TIAI) (idade 65,5 $\pm 5,3$ anos); os participantes eram: idosos com sobrepeso (TIAI, IMC: 
$29,0 \pm 6 \mathrm{~kg} / \mathrm{cm}^{2}$; TAC, IMC: $28,9 \pm 4,2$ $\mathrm{kg} / \mathrm{cm}^{2}$ ), não tabagistas; não portadores de distúrbios respiratórios (DPOC, Asma Brônquica); não usuários de medicação betabloqueadora, ou bloqueadora dos canais de cálcio; não portadores de qualquer doença osteomioarticular ou cardiomiopatia que impossibilitasse o mesmo de executar as atividades; todos os participantes apresentaram liberação médica para a prática de exercício.

Antes de se submeterem aos procedimentos do estudo, os indivíduos responderam uma anamnese e assinaram o termo de consentimento livre e esclarecido. $\mathrm{O}$ presente estudo foi aprovado pelo Comitê de Ética em Pesquisa do Centro Universitário de João Pessoa e está registrado sob protocolo número 50783015.0.0000.5176, cumprindo a Resolução 466/12 do Conselho Nacional de Saúde.

\section{Avaliação da Composição Corporal (ACC)}

Os dados antropométricos de massa corporal, área de gordura visceral, massa livre de gordura, massa de gordura, percentual de gordura, água intracelular, água extra celular, relação cintura quadril e massa mineral óssea foram coletados de acordo com recomendação da Organização Mundial da Saúde através do sistema InBody 720 (8). A estatura foi aferida através de um estadiômetro da
SANNY. As medidas no Inbody foram realizadas antes e após a intervenção de quatro semanas de treinamento.

\section{Protocolo de Medidas da Frequência Cardíaca (FC) e Percepção Subjetiva de Esforço (PSE)}

Para a determinação da frequência cardíaca de treinamento (FCT) e frequência máxima (FCM) foi utilizada a equação proposta por Karvonen et al(9). Para determinação da frequência cardíaca de repouso (FCRep e FCT), foi utilizado um monitor de frequência cardíaca da marca Polar, modelo RS800CX (POLAR ELECTRO OY, KEMPELE, FINLAND). O menor valor de frequência cardíaca registrado no em repouso durante 10 minutos foi considerado como a FCRep.

Para garantir que o exercício fosse realizado dentro FCT, a FC dos indivíduos foi monitorada a cada dez minutos para o TAC e a cada Sprint e intervalo do TIAI, conforme os protocolos. Também foi adotada a escala de percepção subjetiva de esforço (PSE) proposta por. Durante o exercício, a PSE foi questionada estritamente após cada medida de FC. Os índices desta escala refletem a intensidade na qual os sujeitos realizaram o exercício. Para efeito de adequação dos testes, adotamos os valores de 11 a 14 para uma intensidade 
moderada e de 15 a 18 para uma alta intensidade (10).

\section{Protocolo de treinamento Treinamento} Aeróbio Contínuo (TAC)

Realizado em três sessões semanais em cicloergomêtro e de forma continua respeitando também a progressão; o primeiro estágio teve duração de 30 minutos de treinamento com intensidade moderada $(60 \%$ a $80 \%$ da FCres.), no período de quatro semanas. $\mathrm{O}$ segundo do estágio foi realizado no período de três semanas com 40 minutos de exercício em intensidade moderada $(60 \%$ a $80 \%$ da FCres.) e de forma continua respeitando a progressão do treinamento. Por fim, o terceiro estágio, foi realizado com tempo total de execução em 60 minutos entre $60 \%$ a $80 \%$ da FCres, também de forma continua com três semanas de duração. Todo o treinamento foi administrado no período de 10 semanas, totalizando 30 sessões.

\section{Treinamento Intervalado de Alta}

\section{Intensidade (TIAI)}

O treinamento foi realizado em três sessões de cicloergomêtro semanais e em dias alternados, com intensidade controlada entre $85 \%$ a $90 \%$ da FCres, subdividido em três estágios. Estágio um executado em 2 séries de quatro minutos entre $85 \%$ a $90 \%$ da FCres por dois minutos de intervalo ativo a $50 \%$ da FCres totalizando quatro semanas de treinamento. $\mathrm{O}$ estágio dois com três séries de quatro minutos com FCres entre $85 \%$ a $90 \%$, por dois minutos de intervalo ativo a $50 \%$ da FCres, seu período de duração foi de três semanas respeitando a progressão. O último estágio realizado também no período de três semanas, foi executado em quatro séries de quatro minutos de execução $(85 \%$ a $90 \%$ da FCres), por dois minutos de intervalo ativo (50\% da FCres). Essas etapas foram antepostas por um aquecimento de cinco minutos a $50 \%$ da FCres bem como a volta a calma, composta pelo mesmo tempo e percentil do aquecimento.

\section{Análise estatística}

Os dados foram analisados quanto a normalidade (Shapiro-Wilk) e homogeneidade (Levene). Para as análises intragrupos foi o utilizado o teste de Wilcoxon, já nas análises intergrupos foi utilizado o teste de Mann Whitney. Os dados foram analisados no software Graph Pad Prism versão 6.0 sendo considerada diferença significativa para valores de $\mathrm{p} \leq 0,05$.

\section{RESULTADOS E DISCUSSÃO}

Os componentes da composição corporal e suas comparações intra e inter grupos estão apresentadas na figura 1. A área de gordura visceral, apresentou reduções estatisticamente significantes apenas para o grupo TIAI (figura 2). A 
água intra celular, massa mineral óssea, relação cintura quadril e água extra celular estão apresentadas na figura 3.

\section{CONCLUSÃO}

Os achados do presente estudo mostram que 10 semanas de exercício intervalado de alta intensidade aumentou o percentual de massa magra e reduziu à área de gordura visceral em idosos, os demais parâmetros não foram alterados pelo treinamento, diferenças entre $\mathrm{O}$ grupo não foram encontradas sugerindo assim novos estudos para maiores esclarecimento.

\section{REFERÊNCIAS}

1. Silva TCL da, Costa EC, Guerra RO. Resistência aeróbia e força de membros inferiores de idosos praticantes e não-praticantes de ginástica recreativa em um centro de convivência. Rev Bras Geriatr e Gerontol. 2011;14(3):535-42.

2. Little JP, Francois ME. Highintensity interval training for improving postprandial hyperglycemia. Res Q Exerc Sport. 2014 Dec;85(4):451-6.

3. Paz CL, Fraga A, Tenório M. Efeito do treinamento intervalado de alta intensidade versus treinamento contínuo na composição corporal: uma revisão sistemática com meta-análise. Rev Bras Atividade Física Saúde. 2018;22(6):51222.

4. Astorino TA, Heath B, Bandong J, Ordille GM, Contreras R, Montell M, et al. Effect of periodized high intensity interval training (HIIT) on body composition and attitudes towards hunger in active men and women. $\mathbf{J}$
Sports Med Phys Fitness. 2018;58(78):1052-62.

5. de Paiva Montenegro L, Silva de Araújo M, Valle Vieites Novaes E. Influência do treinamento intervalado no controle da obesidade: uma breve revisão. Rev Bras Prescrição e Fisiol do Exerc. 2016;10(61):585-91.

6. Kong Z, Sun S, Liu M, Shi Q. Short-Term High-Intensity Interval Training on Body Composition and Blood Glucose in Overweight and Obese Young Women. J Diabetes Res. 2016;2016:1-9.

7. Mazo GZ, Benedetti TB. Adaptação do questionário internacional de atividade física para idosos. Rev Bras Cineantropometria e Desempenho Hum. 2010;12(6):480-4.

8. Gibson AL, Holmes JC, Desautels RL, Edmonds LB, Nuudi L. Ability of new octapolar bioimpedance spectroscopy analyzers to predict 4component-model percentage body fat in Hispanic, black, and white adults. Am J Clin Nutr. 2008 Feb;87(2):332-8.

9. KARVONEN MJ, KENTALA E, MUSTALA $O$. The effects of training on heart rate; a longitudinal study. Ann Med Exp Biol Fenn. 1957;35(3):307-315.

10. Borg G. Borg's perceived exertion and pain scales. Borg's perceived exertion and pain scales. Champaign, IL, US: Human Kinetics; 1998. viii, 104-viii, 104.

11. Del Vecchio F, Galliano L, Coswig V. Aplicações do exercício intermitente de alta intensidade na síndrome metabólica. Rev Bras Atividade Física Saúde. 2013;18(06).

12. Gibala MJ, McGee SL. Metabolic adaptations to short-term high-intensity interval training: a little pain for a lot of gain? Exerc Sport Sci Rev. 2008 Apr;36(2):58-63. 
13. Khammassi M, Ouerghi N, HadjTaieb S, Feki M, Thivel D, Bouassida A. Impact of a 12-week high-intensity interval training without caloric restriction on body composition and lipid profile in sedentary healthy overweight/obese youth. J Exerc Rehabil. 2018;14(1):11825.

14. Keating SE, Machan EA, O'Connor HT, Gerofi JA, Sainsbury A, Caterson ID, et al. Continuous exercise but not high intensity interval training improves fat distribution in overweight adults. J Obes. 2014;2014:25-7.

15. Binzen CA, Swan PD, Manore MM. Postexercise oxygen consumption and substrate use after resistance exercise in women. Med Sci Sports Exerc. 2001 Jun;33(6):932-8.

16. Foureaux G, Pinto KM de C, Dâmaso A. Efeito do consumo excessivo de oxigênio após exercício e da taxa metabólica de repouso no gasto energético TT - Efecto del consumo excesivo de oxígeno después del ejercicio y de la tasa metabólica de reposo en el desgaste energético TT - Effects of exce. Rev bras med esporte. 2006;12(6):393-8.
Information of this article:

Recebido: 11/04/2020

Aprovado: 21/08/2020

Publicado: 28/12/2020

Received: 11/04/2020

Approved: 21/08/2020

Published: 28/12/2020

Como citar esse artigo / How to cite this article:

Monteiro, E. S. S, et al. Efeito de dez semanas de treinamento intervalado de alta intensidade na composição corporal de idosos. Arq. Bras. Ed. Fís., Tocantinópolis, v. 3, n. 2, Ago./Dez., p. 14 - 19, 2020. 